\title{
Growth and Development of Haemopoietic Cells: A Deterministic Process? *
}

\author{
C.P. Daniel, I.L.O. Ponting and T.M. Dexter
}

\section{A. Introduction}

\section{Growth Factors}

Haemopoietic colony stimulating factors (CSFs) are defined by their ability to stimulate the clonal expansion of bone marrow cells in semi-solid medium. Four main CSFs, interleukin-3 (IL-3), granulocyte macrophage-CSF (GM-CSF), macrophage-CSF (M-CSF) and granulocyte-CSF (G-CSF), have been described. Although all four CSFs are glycoproteins, they are otherwise unrelated. $\mathrm{M}-\mathrm{CSF}$ is the only dimer, the others being monomers with disulphide bridges. Each factor binds to its own distinct receptor which, in the case of M-CSF, is similar to the product of the c-fms oncogene [1]. The responses mediated by these receptors in haemopoietic cells are survival, proliferation, lineage commitment and activation of end cell function.

Each of the four CSFs acts on a different, if overlapping, subset of haemopoietic cells called colony forming cells (CFCs). IL-3 stimulates the differentiation and expansion of a number of different progenitor cell types including $\mathrm{CFC}$ GM, CFC-megakaryocyte, CFC-basophil, and CFC-eosinophil [2]. In addition, when combined with erythropoietin, IL-3 promotes the differentiation and growth of early erythroid progenitors (BFU-E). Colonies formed in the presence of IL-3 may also contain a mix-

Department of Experimental Haematology, Paterson Institute for Cancer Research, Manchester, UK

* This work was supported by the Cancer Research Campaign ture of cell types derived from multipotential cells (CFU-mix) [3]. Thus IL-3 acts both on early, multipotent, cells and on later, lineage committed cell types.

GM-CSF is more lineage restricted than IL-3 although their activities overlap. GM-CSF stimulates the bipotent progenitor cell GM-CFC but can also initiate the growth of BFU-E, CFC-Mix, CFC-Eos and CFC-Meg, although proliferation is not sustained [4]. G-CSF and $\mathrm{M}-\mathrm{CSF}$, as their names imply, are more lineage restricted still $[5,6]$.

In addition to their effects on progenitor cells CSFs can also activate specialised functions in their mature progeny. These include monokine production and phagocytosis in macrophages $[7,8]$, cell mobility in neutrophils [9] and antibody-dependent cytotoxicity in neutrophils and eosinophils [10].

A separate class of haemopoietic factors which are unable to support colony growth have been called synergistic factors [11]. Prominent among these are IL-1 (formerly haemopoietin-1) and IL-4. Both these factors can synergise with CSFs to promote colony growth in vitro. In theory a combination of CSFs and synergistic factors together with lymphokines (not discussed here) could regulate the entire spectrum of cell development from the multipotent progenitor to the activated end cell. In short-term colonyforming assays, however, growth is limited because the progenitor cells on which the growth factors act lack the capacity of self-renewal which is characteristic of normal haemopoiesis. In the bone marrow this capability is supplied by stem cells from which all other lineages are derived. The stem cell can, therefore, be 
defined by its ability to regenerate the entire haemopoietic system including lymphoid cells. In practice, however, it is more usual to assay multipotential cells (CFU-S) by their ability to form colonies in the spleens of potentially lethally irradiated mice [12]. As well as containing all the haemopoietic lineages spleen colonies contain more CFU-S. Thus the CFU-S fulfill many of the criteria applied to stem cells. In order to reproduce these qualities in vitro a different approach to that of the colony forming assay has been adopted. The long-term culture of haemopoietic cells requires that they maintain intimate contact with their stromal microenvironment.

\section{Interactions with Stromal Cells}

Whereas the lifetime of haemopoietic cells in colony-forming assays may be measured in days, those maintained in the presence of a viable adherent layer of bone marrow stromal cells may persist for weeks [13] or even months [14]. Moreover, long-term bone marrow cultures (LTBMC) remain viable in the absence of added growth factors other than serum. Contact between the haemopoietic cells and those of the adherent layer is obviously important. Electron microscopy has shown, for instance, that erythroid progenitors from contacts with macrophages and sinusoidal epithelia. Similarly, granulocytic progenitors are found in contact with reticular cells and preadipocytes. Moreover, separation of the haemopoietic cells from the stroma prevents both self-renewal and differentiation [15].

The importance of stromal contact is further illustrated by experiments with multipotent haemopoietic cell lines (FDCP-Mix). These cells normally depend on the presence of IL-3 for both growth and survival [16]. When seeded onto a layer of marrow-derived stromal cells or mouse embryonic mesenchymal (3T3) cells, however, FDCP-Mix not only survive in the absence of IL-3 but are induced to differentiate. They do not, however, undergo significant self-renewal under these conditions $[17,18]$.

Long-term bone marrow culture and short-term colony forming assays indicate the potential importance of both stroma and growth factors to the regulation of haemopoiesis. Much remains to be discovered, however, about the way this process is actually controlled in vivo. This is especially important in view of the current use of recombinant CSFs as adjuvants in the treatment of various cancers [19]. In this article we attempt to clarify the possible role of growth factors and stromal elements in vivo in the light of recent results from our laboratory.

\section{B. Mechanisms of Stromal Cell Dependent Haemopoiesis}

Both contact with stromal cells and exposure to CSFs can induce progenitor cells to proliferate and differentiate. One mechanism, therefore, by which adherent cells might invoke these responses would be by the production of CSFs. A number of attempts have been made to assay CSF activity in bone marrow adherent cells. M-CSF has been detected by radioimmunoassay but, interestingly, no biological activity could be detected in conditioned medium from adherent cell cultures [20]. This could be due to the secretion of inhibitory substances which mask the activity of the CSF. In contrast, neither GM-CSF nor G-CSF are constitutively produced although both are secreted in response to treatment with IL-1 [21]. There is no evidence that IL-3 is produced by adherent cells.

Conditioned medium from LTBMC does not support the growth of haemopoietic cells which have been enriched for CFU-S [22]. When seeded onto an adherent layer, however, these cells both proliferated and differentiated [23]. This suggests that, if CSFs are present in stroma they must be retained in some way rather than secreted into the medium. Support for this concept is given by the experiments of Gordon et al. [24] who found 
that glycosaminoglycans (GAGs), extracted from bone marrow stromal extracellular matrix, could both bind GMCSF and present it to haemopoietic cells so that it retained its biological activity. It seems possible, therefore, that GAGs could play a role in the retention and presentation of CSFs in stromal cell layers.

Earlier work in our laboratory had shown that modification of proteoglycan synthesis by $\beta$-D-xylosides in LTBMC increased the numbers of haemopoietic cells at all stages of development [25]. Thus it seemed increasingly probable that a component of the extra-cellular matrix (ECM) was important in haemopoiesis. In order to determine which component could be involved in this process, Matrigel, a basement membrane extract containing the various ECM components, was used. Matrigel was found to be capable of binding both GM-CSF and IL-3 and subsequently supporting haemopoietic cells. By selectively degrading the ECM using specific enzymes, it was shown that heparan sulphate was the component responsible for CSF binding [26]. Indeed, heparan sulphate alone, extracted from $3 \mathrm{~T} 3$ cells, is sufficient to bind CSFs and stimulate haemopoietic cells [26]. Direct contact with adherent cells also seems to be important, however, since cell-free ECM does not support haemopoiesis.

These data support the hypothesis that haemopoiesis is regulated in vivo by growth factors produced and presented by the adherent bone marrow cells. We have attempted to confirm this using an in vitro model of haemopoietic differentiation. Factor-dependent FDCP-Mix cells were seeded onto a layer of 3T3 cells under conditions which have been shown to promote differentiation of the haemopoietic cells $[17,18]$. At various times subsequently the RNA from both adherent and haemopoietic cells was probed for the expression of IL-3, GM-CSF and G-CSF by Northern analysis. No mRNA transcripts for any of these CSFs were detected in either cell type. Thus, if stromal-induced haemopoiesis is modulated by these CSFs, they must not only be cryptically retained by the cells, but also produced at very low levels, below the limits of detection for Northern analysis. The possibility that this is perhaps the case is indicated by recent results demonstrating a considerable degree of synergy between growth factors.

\section{Synergistic Interactions}

The direct action of growth factors can only be accurately interpreted by using pure proteins and homogeneous cell populations. We have met the first of these requirements by using recombinant IL-3, GM-CSF and G-CSF and highly purified M-CSF. Previous attempts to produce an enriched stem-cell population have depended on killing cycling cells using 5-fluorouracil (5-FU). This also spares mature cells, however, which are known to produce a number of haemopoietic factors. We have instead used fluorescence-activated cell sorting (FACS) to select a population highly enriched for CFU-Mix cells. FACS-purified cells are about 500 times more enriched for CFUMix than 5-FU cells and can be plated at a low concentration thus eliminating accessory cell interference. Thus, these cells can be used to study the direct effects of factors on multipotent cells.

Using FACS-separated cells we investigated the effects of the synergistic factor IL-1 on colony formation. IL-1 alone had no colony stimulating activity but, in combination with either GM-CSF or M$\mathrm{CSF}$, it induced both more and larger colonies than the CSFs alone. We interpret this to mean that the combination of IL-1 with these factors recruits a population of cells not responsive to the CSFs alone. These cells have a higher proliferation potential, hence the larger colonies, and are therefore probably more primitive than CFU-M or CFU-GM.

In 5-FU-treated bone marrow cells synergy has previously been reported between IL-1 and IL-3 [27]. As discussed 
above, however, 5-FU populations contain accessory cells which may mediate this effect. Alternatively FACS-separated cells may lack a sub-population of primitive progenitors present in the 5-FU population.

The absence of synergy between IL-1 and G-CSF seemed anomalous in view of the effects of IL-1 in combination with other CSFs. In further experiments, however, it was discovered that the spectrum of activity of G-CSF differed from that of the other CSFs. Like IL-1, G-CSF can synergise with M-CSF and GM-CSF to promote increased colony formation. The morphology of the colonies is determined not by G-CSF but by the other factor. Thus, a combination of G-CSF and M-CSF produces mostly macrophage colonies. These data strongly imply that G-CSF and IL-1 act on the same sub-population of cells, a conclusion which was validated by experiments with combinations of three factors. G-CSF and IL-1 plus GM-CSF, M-CSF or IL-3 did not stimulate more proliferation than combinations of two factors. Moreover, the population of cells recruited by IL-1 and G-CSF are probably primitive, IL-3responsive cells. This can be inferred from the observation that the combination of IL-1 plus IL-3 plus M-CSF does not recruit any more cells than either IL-3 alone or IL-3 and IL-1.

While the details of these responses are complex, certain broad principles have emerged from the work. The response of the same population of cells to IL-3, IL-1 and G-CSF suggests that stem cells express receptors for all these factors. They may also have receptors for GM-CSF and M-CSF although these may only be functionally capable of transducing a proliferation response in the presence of IL-1 or G-CSF. Certainly these two factors are capable of recruiting cells to become responsive to GM-CSF and $\mathrm{M}$ CSF. In the light of these findings we propose a model of stem cell development which is deterministic. The primitive multipotential cell has the capacity to respond to a range of factors. The lin- eage it eventually adopts will be determined by the exact combination of factors it encounters. Thus, a stem cell stimulated by IL-1 and M-CSF would give rise to monocytic end cells while one exposed to G-CSF and GM-CSF could produce both monocytes and granulocytes. A combination of factors rather than a single stimulus may be necessary to overcome an internal biochemical threshold which prevents unnecessary proliferation.

The attraction of our proposed model lies in the role it postulates for growth factors in the regulation of multipotent cells. We believe that the early involvement of factors in the determination of lineage commitment allows for both greater flexibility and speed of response than stochastic models. It remains to be seen, however, how far these results obtained in vitro reflect the regulation of haemopoiesis in vivo.

\section{Summary and Conclusions}

The in vitro methods used to study haemopoiesis fall into two distinct categories. Short-term colony forming assays have identified a number of potent soluble factors capable of maintaining survival, proliferation and differentiation of haemopoietic cells but not their selfrenewal. In contrast, in long-term bone marrow culture, extensive self-renewal occurs in the absence of exogenous factors and direct physical contact between haemopoietic cells and cells of the adherent stromal layer seems to be important. Obviously, LTBMC more closely resembles the situation in haemopoietic tissues but the potency of growth factors imply that they too play a role. Our data suggest that this may be at an earlier stage of haemopoietic development than previously appreciated. Primitive multipotent cells have the potential to respond to CSFs which were previously thought to stimulate only committed progenitor cells. This response is only seen, however, when the cells are exposed to a combina- 
tion of factors which include either IL-1 or G-CSF. Thus, a combination of factors is able to recruit cells which are not already committed and determine the lineage along which they will differentiate.

While it remains to be conclusively demonstrated that growth factors regulate normal "steady state" haemopoiesis in vivo it is clear that contact with stromal cells is important. The mechanisms by which the adherent layer influences haemopoietic development, however, are less obvious. We have shown that a component of stroma, heparan sulphate, is able to bind growth factors and present them to haemopoietic cells in a way that stimulates haemopoiesis. The adherent layer, therefore, has the potential to elaborate and present the factors which may control haemopoiesis. It is probably premature to speculate that different classes of adherent cells may present different combinations of factors. It is obvious, however, that the capacity of adherent cells to bind potent haemopoietic factors holds the potential to unify two hitherto separately regarded mechanisms for the regulation of haemopoiesis.

\section{References}

1. Sherr CJ, Rettenmier CW, Sacca R, Roussel MF, Look AT, Ek S (1985) The $c$-fms proto-oncogene product is related to the receptor for mononuclear phagocyte growth factor, CSF-1. Cell 41:665-675

2. Moore MAS (1988) Interleukin 3: an overview. Lymphokines 15:219-228

3. Prystowsky MB, Otten G, Naujokas MF, Vardiman J, Ihle JH, Goldwasser E, Fitch FW (1984) Multiple hemopoietic lineages are found after stimulation of mouse bone marrow precursor cells with interleukin 3 . Am J Pathol 117:171-175

4. Metcalf D, Johnson GR, Burgess AW (1980) Direct stimulation by purified GMCSF of the proliferation of multipotential and erythroid precursor cells. Blood 55: $138-143$

5. Stanley ER, Heard PM (1977) Factors regulating macrophage production and growth. Purification and some properties of the colony stimulating factor from medium conditioned by mouse $\mathrm{L}$ cells. $\mathrm{J}$ Biol Chem 252:4305-4311

6. Nicola NA, Metcalf D, Johnson GR, Burgess AW (1979) Separation of functionally distinct human granulocytemacrophage colony-stimulating factors. Blood 54:614-618

7. Moore RN, Oppenheim JJ, Farrar JJ, Carter CS, Waheed A, Shadduck RK (1980) Production of lymphocyte-activating factor (interleukin 1) by macrophages activated with colony-stimulating factors. J Immunol 125:1302-1308

8. Handman E, Burgess AW (1979) Stimulation by granulocyte-macrophage colony stimulating factor of Leishmania tropica killing by macrophages. J Immunol 122 : 1134-1140

9. Gasson JC, Weisbart RM, Kaufman SE, Clark CS, Hewick RM, Wong GG, Golde GW (1984) Purified human granulocytemacrophage colony-stimulating factor: direct action on neutrophils. Science 226: 1339-1343

10. Vadas MA, Nicola NA, Metcalf D (1983) Activation of antibody-dependent cell-mediated cytotoxicity of human neutrophils and eosinophils by separate colony stimulating factors. J Immunol 130: $795 \mathrm{pp}$.

11. Quesenberry PJ (1986) Synergistic hematopoietic growth factors. Int J Cell Cloning 4:3-15

12. Till JE, McCulloch EA (1961) A direct measurement of the radiation sensitivity of normal mouse bone marrow cells. Radiat Res 14:213-222

13. Dexter TM, Allen TD, Lajtha LG (1977) Conditions controlling the proliferation of haemopoietic stem cells in vitro. J Cell Physiol 91:335-341

14. Dexter TM, Spooncer E, Simmons P, Allen TD (1984) Long-term marrow cultures. An overview of techniques and experience. In: Wright DG, Greenberger JS (eds) Long term bone marrow cultures. Liss, New York

15. Bentley SA (1981) Close range cell:cell interaction required for stem cell maintenance in long-term bone marrow cultures. Exp Hematol 9:308-311

16. Spooncer E, Boettiger D, Dexter TM (1984) Continuous in vitro generation of multipotential stem cell clones from $\mathrm{src}$ infected cultures. Nature 310:228-230

17. Spooncer E, Heyworth CM, Dunn A, Dexter TM (1986) Self-renewal and differentiation of interleukin 3-dependent mul- 
tipotent stem cells are modified by stromal cells and serum factors. Differentiation 31:111-118

18. Roberts RA, Spooncer E, Parkinson EK, Lord BI, Allen TD, Dexter TM (1987) Metabolically inactive 3T3 cells can substitute for marrow stromal cells to promote the proliferation and development of multipotent haemopoietic stem cells. $\mathbf{J}$ Cell Physiol 132: 203-214

19. Bronchud MH, Scarfe JH, Thatcher N, Crowther D, Souza LM, Alton NK, Testa NG, Dexter TM (1987) Phase I/II study of recombinant human granulocyte colonystimulating factor in patients receiving intensive chemotherapy for small cell lung cancer. Br J Cancer 56:809-813

20. Shadduck RK, Waheed A, Greenberger J, Dexter TM (1983) Production of colonystimulating factor in long-term bone marrow cultures. J Cell Physiol 114:88-92

21. Fibbe WE, van Damme J, Billiau A, Goselink HM, Voogt PJ, van Eeden G, Ralph P, Altrock BW, Falkenburg JHF (1988) Interleukin 1 induces human marrow stromal cells in long term culture to produce granulocyte colony-stimulating factor and macrophage colony-stimulating factor. Blood 71:430-436
22. Lord BI, Spooncer E (1986) Isolation of haemopoietic spleen colony forming cells. Lymphokine Res 5:59-63

23. Spooncer E, Lord BI, Dexter TM (1985) Defective ability to self-renew in vitro of highly purified primitive haemopoietic cells. Nature 316:62-64

24. Gordon MY, Riley GP, Watt SM, Greaves MF (1987) Compartmentalization of haematopoietic growth factor (GM-CSF) by glycosaminoglycans in the bone marrow microenvironment. Nature 326: 403-405

25. Spooncer E, Gallagher JT, Krisza F, Dexter TM (1983) Regulation of haemopoiesis in long term bone marrow cultures. IV. Glycosaminoglycan synthesis and the stimulation of haemopoiesis by B-D xylosides. J Cell Biol 96:510-516

26. Roberts R, Gallagher J, Spooncer E, Allen TD, Bloomfield F, Dexter TM (1988) Heparan sulphate bound growth factors: a mechanism for stromal cell mediated haemopoiesis. Nature 332:376-378

27. Stanley ER, Bartocci A, Patinkin D, Rosendaal M, Bradley TR (1986) Regulation of very primitive multipotent cells by hemopoietin-1. Cell 45:667-672 